\title{
The Minimal Clinically Important Difference, Substantial Clinical Benefit, and Patient-Acceptable Symptomatic State after Medial Patellofemoral Ligament Reconstruction
}

\author{
Justin M. Walsh, B.S., Hailey P. Huddleston, M.D., Mohamad M. Alzein, B.S., \\ Stephanie E. Wong, M.D., Brian Forsythe, M.D., Nikhil N. Verma, M.D., \\ Brian J. Cole, M.D., M.B.A., and Adam B. Yanke, M.D., Ph.D.
}

\begin{abstract}
Purpose: To define the minimal clinically important difference (MCID), substantial clinical benefit (SCB) and patientacceptable symptomatic state (PASS) for patient-reported outcome measures (PROMs) after medial patellofemoral ligament reconstruction (MPFLR) and to investigate the role of preoperative, demographic, and intraoperative variables for predicting achievement of these thresholds. Methods: This retrospective cohort study used a prospectively maintained database of patients undergoing primary MPFLR between August 2015 and December 2019. PROMs included the International Knee Documentation Committee (IKDC), Knee Injury and Osteoarthritis Outcome Score (KOOS), KOOS joint replacement (JR), and Kujala. Anchor-based and distribution-based methods were used to calculate the MCID, SCB, and PASS. Regression analyses were performed to identify prognosticators for achievement of clinically significant thresholds. Results: 139 patients met inclusion criteria (mean age: $21.7 \pm 8.2$ years). At 6 months, the MCID values were 8.3 (KOOSPain) and 8.5/13.5 (Kujala); SCB values were 1.4 (KOOS-Pain) and 43.7 (KOOS-QOL); and PASS values were 64.9 (IKDC), 83.3 (KOOS-Symptom), 76.8 (KOOS-Pain), 91.2 (KOOS-ADL), 47.5 (KOOS-Sport), 40.6 (KOOS-QOL), and 78.1 (KOOSJR). At 1 year, the MCID values were 4.2 (KOOS-Pain), 7.2 (KOOS-ADL), 12.4 (KOOS-QOL) and 25.2 (KOOS-JR); SCB were 23.6 (IKDC), 4.2 (KOOS-Symptom), 19.7 (KOOS-Pain), 6.5 (KOOS-ADL), 55.0 (KOOS-Sport), 6.3 (KOOS-QOL), and 19.6/25.2 (KOOS-JR); and PASS were 65.5 (IKDC), 80.4 (KOOS-Symptom), 84.7 (KOOS-Pain), 99.3 (KOOS-ADL), 57.5 (KOOS-Sport), 53.1 (KOOS-QOL), and 76.3 (KOOS-JR). In regression analysis, greater age, body mass index, and preoperative PROMs were negative prognosticators for achieving clinically significant thresholds. Conversely, male gender increased the likelihood of achieving PASS for Kujala at 6 months and KOOS-ADL at 1 year. Conclusions: This study established thresholds for the MCID, SCB, and PASS at 6 months and 1 year after MPFLR, providing physicians an evidence-based method to advise patients and assess outcomes with this surgery. Older patients and those with higher preoperative outcome scores are less likely to report improvement and satisfaction with MPFLR, while male patients are more likely to report some satisfaction. Level of Evidence: Level III, retrospective cohort study (diagnosis).
\end{abstract}

From Rush University Medical Center, Chicago, Illinois, U.S.A.

The authors report the following potential conflicts of interest or sources of funding: S.E.W. reports being a paid speaker for DJ Orthopaedics and has received grants from Arthrex, Inc. and Acumed. B.F. reports personal fees from Elsevier, Jace Medical, and Stryker and being a paid consultant for Stryker, outside the submitted work. A.B.Y. reports grants from Organogenesis, Vericel, and Arthrex, Inc.; personal fees from CONMED Linvatec, JRF Ortho, and Olympus; nonfinancial support from Patient $I Q$, Smith $\theta$ Nephew, Sparta Biomedical; and stock or stock options from Patient IQ, outside the submitted work. N.V. reports receiving personal fees from Cymedica, Minivasive, Omeros, Orthospace, Stryker, and Medacta USA, Inc.; receiving royalties from Arthroscopy, Smith $\theta$ Nephew, and Vindico MedicalOrtho Hyperguide; being a board or committee member of AOSSM, ASES, AANA, Knee, and SLACK, Inc., outside the submitted work. B.J.C. reports personal fees from Ossio, Regentis, and Arthrex; research support from the National Institutes of Health and Aesculap; being a paid consultant for
Aesculap, Regentis, Arthrex, Acumed, and Vericel Corporation; IP royalties from Elsevier, Arthrex, and Operative Techniques in Sport Medicine; and stock or stock options from Ossio, Regentis, and Bandgrip, Inc., outside the submitted work. Full ICMJE author disclosure forms are available for this article online, as supplementary material.

Received April 21, 2021; accepted December 12, 2021.

Address correspondence to Adam B. Yanke, M.D., Ph.D., Rush University Medical Center, 1611 W. Harrison St., Chicago, IL 60612, U.S.A. E-mail: adam.yanke@rushortho.com

(C) 2022 Published by Elsevier Inc. on behalf of the Arthroscopy Association of North America. This is an open access article under the CC BY-NC-ND license (http://creativecommons.org/licenses/by-nc-nd/4.0/).

2666-061X/21537

https://doi.org/10.1016/j.asmr.2021.12.009 


\section{Introduction}

$\mathbf{P}$ atient-reported outcome measures (PROMs) are used in clinical research to provide snapshots of patients' levels of pain, function, activity level, and general quality of life. ${ }^{1}$ One limitation of PROMs is that outcomes have typically been reported in terms of statistical significance, which may not represent clinical relevance or outcomes that patients perceive as important. With a growing focus in medicine on patient-centered outcomes and defining meaningful patient-reported improvement, clinically significant outcomes are increasingly emphasized over ones solely defined by statistical significance to demonstrate clinical benefit. $^{1-3}$

Three metrics for clinical significance have been developed to aid in the understanding of outcome scores at the individual patient level. These include the minimal clinically important difference (MCID), the substantial clinical benefit (SCB), and the patientacceptable symptomatic state (PASS). The MCID is defined as the smallest clinical improvement that the patients perceive as important. ${ }^{2,3}$ The SCB establishes a threshold that patients determine to be a considerable improvement. ${ }^{2,3}$ Lastly, the PASS represents the postoperative outcome score associated with achieving patient satisfaction. ${ }^{3,4}$ These measures represent a stratification of postoperative outcomes in which MCID represents a minimal improvement, SCB represents optimal improvement, and PASS represents patient satisfaction with their outcome. ${ }^{4}$ Defining these values for specific procedures gives physicians a reliable, evidence-based method to measure and track clinical improvements postoperatively, as well as to help guide clinical decision making. ${ }^{3}$

The medial patellofemoral ligament (MPFL) is the primary restraint against lateral translation of the patella and is often injured in patellar dislocations. ${ }^{5-7}$ Initial treatment for patellar instability and/or subluxation often includes conservative management, consisting of physical therapy and bracing of the knee joint. Recurrent instability often requires surgical intervention, and MPFL reconstruction has become an increasingly popular treatment option..$^{7-9}$ However, the thresholds for MCID, SCB, and PASS for MPFL reconstruction have yet to be defined in the literature. Appropriately defining these values can provide better understanding of PROMs and clinical success after MPFL reconstruction. Therefore, the goal of this study was to define the MCID, SCB, and PASS for PROMs after MPFLR and to investigate the role of preoperative, demographic, and intraoperative variables for predicting achievement of these thresholds. The hypothesis of this study was that there are net changes and absolute outcome score values that represent the MCID, SCB, and PASS that can accurately predict patient perception of improvement and satisfaction, and, additionally, that there are patient variables that can be used to predict achievement of these thresholds.

\section{Materials and Methods}

\section{Study Design}

This retrospective cohort study was approved by the Institutional Review Board at Rush University Medical Center with a corresponding waiver of consent. A query of a prospectively maintained institutional database was conducted for all patients who underwent MPFL reconstruction by the four senior authors B.F., N.N.V., B.J.C., and A.B.Y. between August 2015 and December 2019 with a minimum of a 6-month follow-up. A 6-month follow-up was used to align with the 4-to-6-month recovery that is typical for MPFL reconstruction. ${ }^{10}$ Any patients in the database who completed preoperative PROMs were included in the study. MPFL reconstruction was the primary procedure for all patients in the cohort, and concomitant procedures are detailed in Table 1 . Exclusion criteria included revision MPFL reconstruction and more significant concomitant procedures, including osteochondral allograft (OCA), osteochondral autograft (OAT), autologous chondrocyte implantation (ACI), matrix-induced chondrocyte implantation (MACI), tibial tubercle osteotomy (TTO), meniscus repairs and transplants, and ligamentous reconstructions. Patients were similarly excluded if they failed to complete their preoperative patient-reported outcome questionnaires. Patients with prior MPFL reconstruction were excluded, as this was considered a revision procedure; however, prior MPFL repair was not an exclusion criterion. Additionally, given the high prevalence of cartilage damage caused by patellar dislocations, which has been reported to be as high as $40-96 \%$ by MRI studies, ${ }^{11}$ it was not feasible to exclude patients with mild or low-grade chondral defects from the current study.

\section{Patient Assessment and Patient Reported Outcome Measures}

Outcome scores examined in this study included the IKDC, KOOS, KOOS JR, and Kujala scores. ${ }^{12-15}$ The IKDC is an evaluation used to measure symptoms, function, and sports activity for people with a variety of knee disorders, while the Kujala is a scale designed to evaluate anterior knee pain. The KOOS is subdivided into 5 separate subscales: Pain, Symptoms, Activities of Daily Living (ADL), Sport, and knee-related Quality of Life (QOL). All patients were assessed preoperatively and at 6 months postoperatively. A subset of patients in this cohort who were at least 1 year but no greater than 18 months from date of surgery were also assessed at 1 year postoperatively. Patient outcome questionnaires were initially administered via email, and follow-up for nonresponders was conducted using email and phone 
Table 1. Concomitant Procedures

\begin{tabular}{lccc}
\hline & $\begin{array}{c}\text { Not Missing } \\
\text { Anchor } \\
\text { Questions }\end{array}$ & $\begin{array}{c}\text { Missing } \\
\text { Anchor } \\
\text { Questions }\end{array}$ & $\begin{array}{c}\text { Total } \\
\text { Patients }\end{array}$ \\
\hline Concomitant Procedures & & & \\
Partial lateral Meniscectomy & 1 & 1 & 2 \\
Microfracture & 0 & 2 & 2 \\
Chondroplasty & 34 & 44 & 78 \\
Synovectomy & 0 & 3 & 3 \\
Bursa excision & 0 & 1 & 1 \\
Loose body removal & 13 & 11 & 24 \\
ACI biopsy & 3 & 4 & 7 \\
Plica excision & 0 & 1 & 1 \\
Trochlear bumpectomy & 1 & 0 & 1 \\
\hline
\end{tabular}

ACI, autologous chondrocyte implantation.

calls. Patients were deemed not available for follow-up if PRO questionnaires were not completed by 9 months postoperatively for the 6-month time point, and 18 months postoperatively for the 1-year time point. Furthermore, we collected clinical history and demographic variables, including age, sex, body mass index, workers' compensation status, smoking history, laterality, age at first instability event, and total number of instability events.

\section{Anchor Questions}

Patients received two anchor questions at 6 months and 1 year postoperatively. These questions were used as anchors to determine clinically meaningful changes in outcomes. ${ }^{4}$ The approach outlined by Juniper et al. ${ }^{16}$ was used to determine the MCID and SCB. For the KOOS, six global questions (related to each of the KOOS subscales) were used to classify patients based on whether they had improved, had no change, or had deteriorated since the previous clinic visit. The questions were phrased, "Since your last surgery, has there been any change in your pain/symptoms/activities of daily living/sport and recreation/quality of life/overall activity level as it is related to your knee?". As the Kujala and KOOS JR scores both consist of questions across multiple domains, they were each assessed against multiple anchor questions. In this study, the knee function and symptoms anchors were selected to correspond with the Kujala score, as they had the best fit with the domains covered by the Kujala score. Additionally, the physical function and symptom domains appear to be relevant to our young, active population. Knee-related pain, symptoms, and activities of daily living were selected to correspond with the KOOS JR to match the three domains covered by this scale. For the IKDC, patients were asked the global question: "Since your last surgery, has there been any change in the overall function of your knee?".

If patients indicated there was no change, they were given a score of 0 . If there was a change between follow-up visits, they were asked to quantify that change on the following scale: a very great deal worse $(-7)$, a great deal worse $(-6)$, a good deal worse $(-5)$, moderately worse $(-4)$, somewhat worse $(-3)$, a little worse $(-2)$, almost the same, hardly any worse at all $(-1)$, no change $(0)$, almost the same, hardly any better at all (1), a little better (2), somewhat better (3), moderately better (4), a good deal better (5), a great deal better (6), or a very great deal better (7).

The anchor question designed by Tubach et al. ${ }^{17}$ was used to determine the PASS for all the subscales of the KOOS, IKDC, KOOS JR, and Kujala scores. At each follow-up, patients were asked the following binomial question: "Taking into account all the activities you have during your daily life, your level of pain, and also your functional impairment, do you consider that your current state is satisfactory?" Anchor questions are summarized in Fig 1.

\section{Surgical Technique and Rehabilitation Protocol}

The preferred technique for MPFL reconstruction used by the senior authors in this study has been previously described and published by Anbari et al. ${ }^{18}$ Associated pathologies, including minor soft tissue and cartilaginous defects, were treated concomitantly when present. All MPFL reconstructions were performed with a double-limbed patellar anchor technique, as outlined by previously published studies. ${ }^{18-20}$ Semitendinosus or tibialis anterior allografts were used for the reconstruction based upon surgeon preference.

Postoperative rehabilitation followed a previously described protocol, ${ }^{18}$ with 6 weeks of protected weightbearing with heel slides, quadriceps and hamstring sets, patellar mobilizations, and gastrocnemius stretches. The brace was unlocked upon regaining quadriceps strength. At 6 weeks, the brace was discontinued and patients were progressed to full weightbearing flexion exercises, toe raises, and closed chain quadriceps work. In-line jogging and elliptical exercises were permitted at 12 weeks under PT supervision, and progression to sports-specific exercises and return to play was permitted by 16 weeks postoperatively.

\section{Determination of Clinically Significant Thresholds}

The MCID can be reliably ascertained with the distribution-based method by calculating $50 \%$ of the standard deviation (SD) for change between preoperative and postoperative scores for a given PROM. The $50 \%$ SD has previously been shown to be a reliable threshold for important patient-perceived change. ${ }^{21}$ Anchor-based thresholds are typically calculated by first stratifying the patients' subjective feelings of improvement into three tiers: no change, minimal improvement, and substantial improvement. The thresholds are then determined by comparing the changes in mean PROM scores between the classification groups. The corresponding difference between the 
A Anchor Question: Since your last surgery, has there been any change in your pain/symptoms/activities of daily living/sport and recreation/quality of life/overall activity level as it is related to your knee?

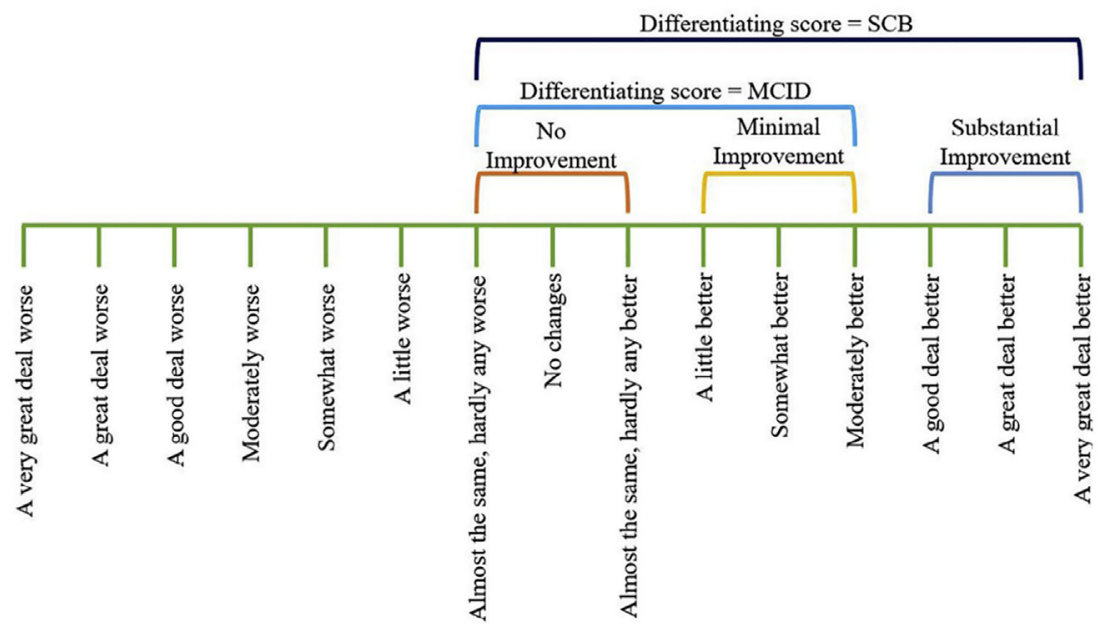

Fig 1. Anchor-based calculation of minimally clinically important difference (MCID) and substantial clinical benefit (SCB) (A), and patientacceptable symptom state (PASS) (B). ${ }^{4}$

B Anchor Question: Taking into account all the activities you have during your daily life, your level of pain, and also your functional impairment, do you consider that your current state is satisfactory?

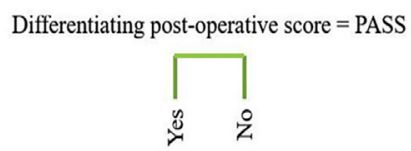

"no change" and "minimal improvement" groups was used to define the MCID, and the mean difference between the "no change" and "substantial improvement" groups was used to define SCB. The PASS threshold was calculated in an identical manner with absolute postoperative values at 6 months and 1 year, using the anchor question regarding satisfaction to differentiate and compare satisfied patients from unsatisfied patients. $^{1,22}$

\section{Statistical Analysis}

To assess for potential selection bias, age, sex, body mass index (BMI), and preoperative outcome scores were compared between patients who completed the minimum 6-month follow-up and those patients who were not available for follow-up, using a two-sample Student's $t$-test and $\chi^{2}$ test for continuous and categorical variables, respectively. Nonparametric receiver operating characteristic (ROC) curves with area under the curve (AUC) analyses were used to evaluate each outcome score to predict the MCID change, SCB change, and PASS based on the above anchor method calculation. The Youden Index was used with the ROC analysis to identify the threshold value which optimized sensitivity and specificity for each outcome score. ${ }^{16}$ Consistent with prior studies, ${ }^{1,3,4}$ the degree of association was acceptable if the AUC was greater than .7, and excellent if the AUC was greater than .8. Threshold analyses for all PROMs were subsequently performed for all patients to determine which achieved the MCID, SCB, or PASS. Secondary analyses using univariate and multivariate regressions were performed to assess whether individual patient factors were correlated with achieving MCID, SCB, or PASS. Univariate logistic regression analyses were performed with respect to each patient variable, using the $\chi^{2}$ test for categorical variables or the Student's $t$ test for continuous variables. Age, BMI, age at first instability event, total number of instability events, and preoperative PRO scores were analyzed as continuous variables. Sex and workers' compensation status were analyzed as categorical variables. Variables were subsequently included in multivariate regression analysis if univariate analysis found significance of $P<.10$. The odds ratio (OR) and 95\% confidence interval (CI) were determined for each variable with respect to achieving MCID, SCB, and PASS. An a priori power analysis was conducted based on a similar study establishing clinically significant 
Table 2. Patient Demographic and Clinical Variables of Included Patients

\begin{tabular}{lc}
\hline \multicolumn{1}{c}{$\mathrm{n}$} & 139 \\
\hline Age (years) & $21.7 \pm 8.2$ \\
Sex & \\
$\quad$ Male & $49(35.0 \%)$ \\
$\quad$ Female & $90(65.0 \%)$ \\
BMI $\left(\mathrm{kg} / \mathrm{m}^{2}\right)$ & $25.8 \pm 5.9$ \\
WC & $7(5.0 \%)$ \\
Smoker & $15(13.0 \%)$ \\
Previous surgery on index knee & $29(20.9 \%)$ \\
MPFL Repair & $8(5.8 \%)$ \\
Laterality & \\
$\quad$ Left & $90(65.0 \%)$ \\
$\quad$ Right & $49(35.0 \%)$ \\
IKDC & $47.3 \pm 18.2$ \\
KOOS symptom & $63.4 \pm 17.8$ \\
KOOS pain & $66.2 \pm 18.5$ \\
KOOS ADL & $75.4 \pm 18.3$ \\
KOOS sport & $36.6 \pm 26.0$ \\
KOOS QOL & $25.9 \pm 18.0$ \\
KOOS JR & $66.0 \pm 14.6$ \\
Kujala & $57.8 \pm 18.3$ \\
\hline
\end{tabular}

Data are presented as means \pm SD or $n(\%)$ for countable variables. $\mathrm{ADL}$, activities of daily living; BMI, body mass index; IKDC, International Knee Documentation Committee; JR, joint replacement; KOOS, Knee Injury and Osteoarthritis Outcome Score; QOL, quality of life; TTO, tibial tubercle osteotomy; WC, Workers Compensation.

thresholds following meniscal allograft transplantation. ${ }^{1,3}$ Using the mean change from baseline and the MCID for each outcome score, we calculated the effect size (mean $=.266$; range: .021-.513), which was used to determine an appropriate sample size. Using $G^{*}$ power, a total sample size of 89 was deemed sufficient for analysis, and a cohort of 100 patients was chosen to slightly enhance the power of the study. All other statistical analyses were performed using SPSS, version 26.0 (IBM, New York), and assumed a significance level of .05.

\section{Results}

\section{Patient Demographics}

A total of 139 patients met inclusion criteria $(90$ females [65.0\%] and 49 males [35.0\%], mean age: 21.7 \pm 8.2 years, mean BMI: $25.8 \pm 5.9)$, with 83 included for analysis at 6 months and 56 included at 1 year (Appendix Fig. 1). Seven (5.0\%) patients had workers' compensation status, and $15(13.0 \%)$ were current or former smokers. $20.9 \%$ of patients reported previous surgeries on the index knee prior to undergoing MPFL reconstruction. Demographic data and preoperative PROM scores are summarized in Table 2. Patients who responded to the anchor questions did not differ significantly from nonresponders (Table 3 ).

\section{Patient-Reported Outcomes}

Of the 139 patients in the cohort, 108 (77.7\%) patients provided responses to the anchor questions. The baseline scores are shown in Table 2. Demographic and preoperative clinical outcomes between responders and nonresponders were not found to be statistically different from each other (Table 3). Concomitant procedures are summarized in Table 1.

\section{Calculating Clinically Significant Outcomes (MCID, SCB, and PASS)}

Change in PROM at 6-month and 1-year from baseline values are shown in Appendix Table 1. From these, the distribution-based MCID, SCB, and PASS thresholds at 6 months and 1 year were calculated (Tables 4, 5, and 6). The anchor-based approach was used if AUC was acceptable (>.7); otherwise, the distribution-based thresholds were implemented. A concise summary of the calculated thresholds is shown in Table 7.

\section{Variables Associated with Clinical Significance}

Preoperative patient demographic data were analyzed to determine whether any factors were associated with achieving calculated MCID, SCB, and PASS thresholds. Univariate and multivariate analyses demonstrated that greater age is associated with reduced odds of achieving MCID for IKDC and Kujala (vs symptom), SCB for Kujala (vs symptom), and PASS for KOOS Symptom, Pain, and ADL at 6 months. Higher BMI was associated with reduced odds of achieving PASS for IKDC at 1 year. Male sex increased the odds of achieving PASS for Kujala at 6 months and for KOOS ADL at 1 year. Lastly, higher preoperative PRO scores were associated with a reduced likelihood of achieving MCID, SCB, and PASS for multiple PROMs at both time points. Logistic regression results are summarized in Appendix Tables 2, 3, and 4 .

\section{Discussion}

The present study established values for the MCID, SCB, and PASS at 6 months and 1 year postoperatively for patients undergoing primary MPFL reconstruction with respect to commonly administered knee and patellofemoral outcome measures, including IKDC, Kujala, KOOS JR, and KOOS subscales (Pain, Symptoms, ADL, Sport, and QOL) (Table 7). We chose the 6month follow-up over a later time point to align with the 4-to-6-month return to sport timetable that is commonly used for rehabilitation following MPFL reconstruction, according to previously published protocols. ${ }^{10}$ Additionally, earlier timepoints have been previously shown to be more useful for calculating the MCID using the anchor-based method, because the relatively smaller percentage of patients falling into the "no change" group at later time points lends a negative influence on the power of the calculations. ${ }^{3,23}$ Finally, 
Table 3. Comparison of Responders and Nonresponders on Anchor Questions

\begin{tabular}{|c|c|c|c|}
\hline & Not Missing Anchor Questions $(n=108)$ & Missing Anchor Questions $(n=31)$ & $P$ Value \\
\hline$\overline{\text { Age (years) }}$ & $22.2 \pm 8.8$ & $20.7 \pm 6.7$ & .29 \\
\hline Sex, M:F & $39: 69$ & 10:21 & .16 \\
\hline BMI & $25.5 \pm 6.0$ & $26.3 \pm 5.5$ & .46 \\
\hline Smoker & $14(13.0 \%)$ & $1(3.2 \%)$ & .19 \\
\hline Laterality, L:R & $71(65.7 \%): 37(34.3 \%)$ & $29(61.3 \%): 12(38.7 \%)$ & .62 \\
\hline IKDC & $48.0 \pm 18.2$ & $45.8 \pm 18.3$ & .53 \\
\hline KOOS ADL & $76.8 \pm 17.4$ & $73.9 \pm 19.3$ & .45 \\
\hline KOOS Sport & $35.9 \pm 26.1$ & $37.3 \pm 26.2$ & .80 \\
\hline KOOS QOL & $27.1 \pm 19.8$ & $24.7 \pm 16.2$ & .53 \\
\hline KOOS JR & $66.7 \pm 14.4$ & $64.3 \pm 15.0$ & .38 \\
\hline Kujala & $57.9 \pm 18.1$ & $57.5 \pm 19.7$ & .92 \\
\hline
\end{tabular}

ADL, activities of daily living; BMI, body mass index; IKDC, International Knee Documentation Committee; JR, joint replacement; KOOS, Knee Injury and Osteoarthritis Outcome Score; QOL, quality of life; WC, Workers Compensation.

because of the relative paucity of the literature on the topics of postoperative outcomes and recovery timetables after MPFL reconstruction, we also wanted to allow for analysis of both 6-month and 1-year postoperative data to help fill these gaps in the literature.

The three commonly accepted strategies used to determine the MCID include "anchor-based", "distribution-based" or "opinion-based" methodologies. ${ }^{16,24}$ The United States Food and Drug Administration's (FDA) final guidelines to industry when evaluating effectiveness of treatment using PROMs recommends the anchor-based approach to demonstrate treatment benefit. $^{25}$ The anchor-based approach allows for the comparison of changes in PROMs against anchor questions that assess subjective improvement in global domains such as overall symptoms or function. ${ }^{16,24,26}$ The distribution-based method uses statistical analysis to determine the minimal clinically significant change for a given PROM, but has only been validated for use with calculating MCID. ${ }^{1,21}$ In the present study, anchor-based and distribution-based methods were both used to determine MCID to enhance predictive power, and only the anchor-based method was used to determine SCB and PASS. Although some studies have argued for the use of both anchor- and distributionbased methods to determine MCID, ${ }^{1,27}$ one drawback is the difficultly in explaining discrepancies that may arise. Several inconsistencies were observed in the current study, including the MCID and SCB for multiple PROMs, which may be attributed to the low predictive power of these values, as evidenced by their unacceptable AUCs. In these cases, the distribution-based scores were used to define MCID, as the low AUCs suggested the anchor-based values were unreliable. By minimizing the use of thresholds that have unacceptable AUCs, we were able to maximize the predictive power of these models and reduce potential bias.
Using the calculated threshold values, we found several factors associated with failing to achieve these clinically significant outcomes following primary MPFL reconstruction, including advanced age at time of surgery, higher preoperative PRO scores, and higher BMI. Conversely, we found that male gender was associated with a greater likelihood of achieving PASS for Kujala at 6 months, and KOOS Symptom and ADL at 1 year. These threshold values are important in providing physicians and patients with benchmarks for postoperative recovery as well as insight into the changes in PROM scores necessary to achieve improvements after MPFL reconstruction that are significant and acceptable to the patient.

The use of MPFL reconstruction to treat recurrent patellar instability has gained much popularity in recent years, owing to its effectiveness in restoring the medial restraining forces of the patella $a^{6,7}$ and positive clinical outcomes. $^{28,29}$ A 2016 systematic review and metaanalysis of 14 studies reported an average postoperative Kujala score of 85.8 with an $84.1 \%$ return to sport rate following isolated MPFL reconstruction in the setting of chronic patellar instability, as well as low incidence of recurrent instability, postoperative apprehension, and revision surgery in the short-term. ${ }^{28}$ Sappey-Marinier et $\mathrm{al}^{29}$ reported a similar improvement in Kujala following isolated MPFL reconstruction, with patients improving from an average score of 56.1 preoperatively to 88.8 postoperatively. These authors also found that over $84 \%$ of patients returned to sport at the same level of performance. In the current study looking at MPFL reconstructions performed in isolation and with minor concomitant procedures, we found that Kujala improved from a baseline of 57.8 to 82.8 at 6 months and to 86.5 at 1 year postoperatively. Overall, we found that the change from baseline improved for all PROMs from 6 months to 1 year. This improvement 
Table 4. MCID Values at 6 Months and 1 Year

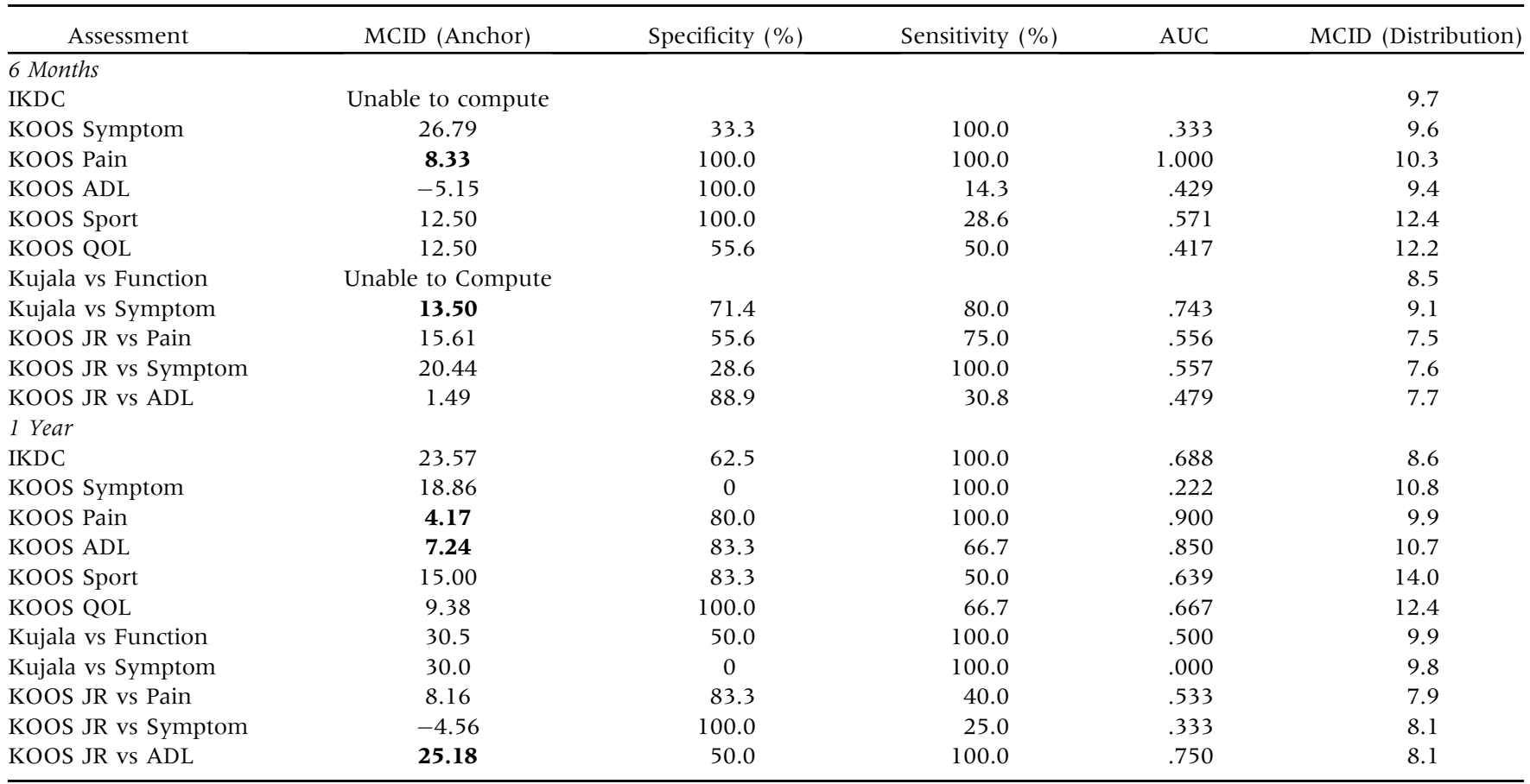

Bold indicates AUC > .7. ADL, activities of daily living; AUC, area under the curve; IKDC, International Knee Documentation Committee; JR, joint replacement KOOS, Knee Injury and Osteoarthritis Outcome Score; MCID, minimal clinically important difference; QOL, quality of life.

Table 5. SCB Values at 6 Months and 1 Year

\begin{tabular}{|c|c|c|c|c|}
\hline Assessment & SCB (Anchor) & Specificity $(\%)$ & Sensitivity (\%) & AUC \\
\hline \multicolumn{5}{|l|}{6 Months } \\
\hline IKDC & Unable to Compute & & & \\
\hline KOOS Symptom & 14.29 & 55.9 & 100.0 & .559 \\
\hline KOOS ADL & 25.74 & 38.5 & 85.7 & .569 \\
\hline KOOS Sport & 47.50 & 39.1 & 87.5 & .596 \\
\hline KOOS QOL & 43.66 & 52.0 & 100 & .735 \\
\hline KOOS JR vs Pain & 21.43 & 34.0 & 100.0 & .559 \\
\hline KOOS JR vs Symptom & 21.43 & 30.2 & 100.0 & .653 \\
\hline KOOS JR vs ADL & 23.52 & 28.2 & 92.3 & .579 \\
\hline \multicolumn{5}{|l|}{1 Year } \\
\hline IKDC & 23.56 & 80.0 & 100.0 & .886 \\
\hline KOOS Symptom & 4.17 & 83.3 & 100.0 & .917 \\
\hline Kujala vs Function & 30.0 & 53.6 & 100.0 & .554 \\
\hline Kujala vs Symptom & 30.0 & 55.2 & 100.0 & .600 \\
\hline KOOS JR vs Pain & 19.62 & 60.6 & 100.0 & .761 \\
\hline KOOS JR vs Symptom & 27.05 & 38.5 & 100.0 & .638 \\
\hline KOOS JR vs ADL & 25.18 & 46.9 & 100.0 & .758 \\
\hline
\end{tabular}

Bold indicates AUC > .7; AUC, area under the curve; ADL, activities of daily living; IKDC, International Knee Documentation Committee; KOOS, Knee injury and Osteoarthritis Outcome Score; JR, joint replacement; QOL, quality of life; SCB, substantial clinical benefit. 
Table 6. PASS Values at 6 Months and 1 Year

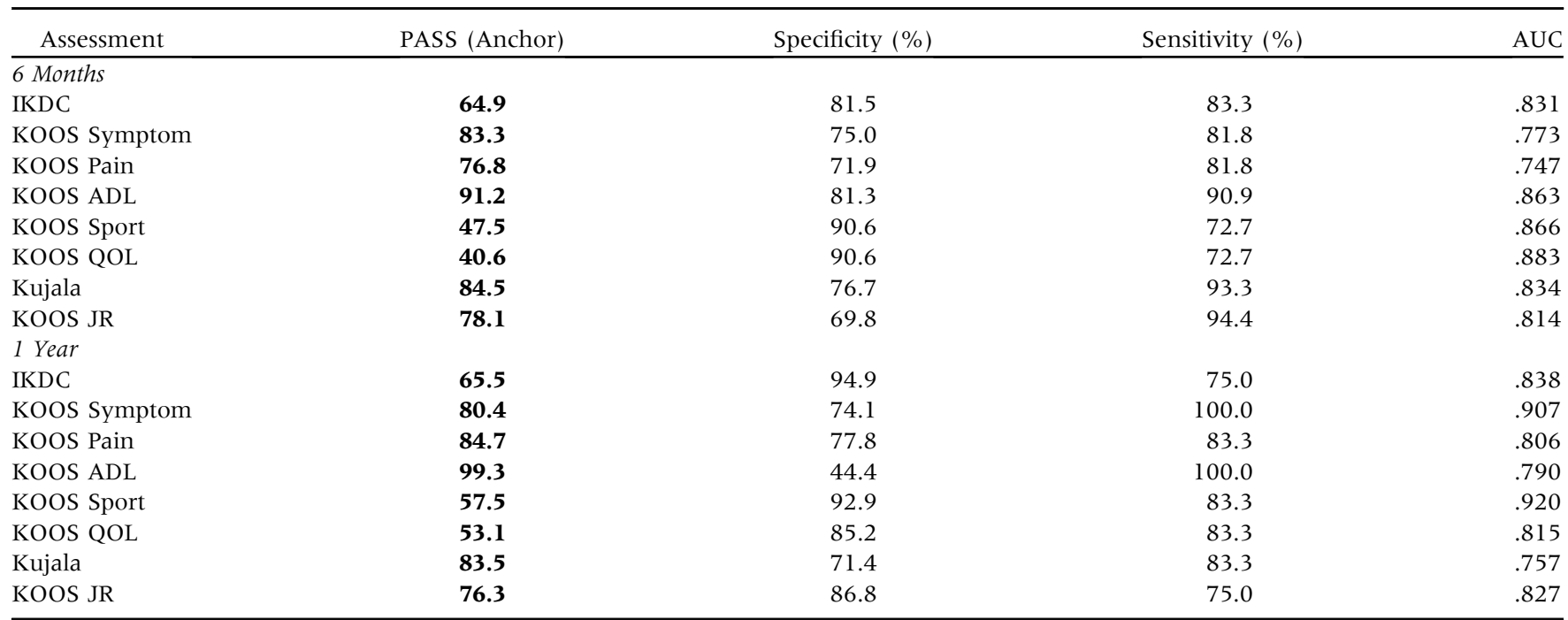

Bold indicates AUC > 0.7. ADL, activities of daily living; AUC, area under the curve; IKDC, International Knee Documentation Committee; JR, joint replacement KOOS, Knee Injury and Osteoarthritis Outcome Score; PASS, patient-acceptable symptomatic state; QOL, quality of life.

aligns with the 4-to-6-month recovery timetable previously published, ${ }^{10}$ and continues up to 1 year after surgery. Outcomes beyond 1 year are beyond the scope of the current study, and further investigation using longer follow-up timeframes is warranted.

Within MPFL reconstruction surgery, the importance of preoperative and demographic variables associated with postoperative outcomes is well recognized. ${ }^{29-32}$ In our study, we found that older age at time of surgery was associated with a decline in postoperative outcomes, carrying a reduced odds ratio of achieving MCID, SCB, and PASS for multiple PROMs at 6 months. We also found that higher BMI at the time of surgery exerted a negative influence on achieving PASS for IKDC at 1 year. These results corroborate previous literature highlighting the negative influence of age on postoperative outcomes. ${ }^{30}$ Interestingly, however, we found that male sex positively influenced the odds of achieving PASS for Kujala at 6 months, and for KOOS Symptom and ADL at 1 year, which contradicts prior studies that found sex to be noninfluential on postoperative outcomes. ${ }^{30}$

Lastly, we found that higher preoperative scores were negative prognosticators for achieving MCID and SCB but slightly positively influenced the achievement of PASS for KOOS QOL at 6 months, which corroborates an existing trend in the literature. ${ }^{31-34}$ This is fairly intuitive because patients with greater symptoms and impairment before surgery have more room for improvement and, thus, are likely to achieve a greater

Table 7. Summary of MCID, SCB, and PASS Threshold Values at 6 Months and 1 Year

\begin{tabular}{|c|c|c|c|c|c|c|}
\hline & \multicolumn{2}{|c|}{ MCID } & \multicolumn{2}{|c|}{ SCB } & \multicolumn{2}{|c|}{ PASS } \\
\hline & 6 Months & 1 Year & 6 Months & 1 Year & 6 Months & 1 Year \\
\hline IKDC & 9.7 & 8.6 & & 23.6 & 64.9 & 65.5 \\
\hline KOOS Pain & 8.3 & 4.2 & 1.4 & 19.7 & 76.8 & 84.7 \\
\hline KOOS ADL & 9.4 & 7.2 & 25.7 & 6.5 & 91.2 & 99.3 \\
\hline KOOS Sport & 12.4 & 14.0 & 47.5 & 55.0 & 47.5 & 57.5 \\
\hline KOOS QOL & 12.2 & 12.4 & 43.7 & 6.3 & 40.6 & 53.1 \\
\hline KOOS JR vs pain & 7.5 & 7.9 & 21.4 & 19.6 & 78.1 & 76.3 \\
\hline KOOS JR vs symptom & 7.6 & 8.1 & 21.4 & 27.1 & & \\
\hline KOOS JR vs ADL & 7.7 & 25.2 & 23.5 & 25.2 & & \\
\hline
\end{tabular}

Bold indicates AUC > .7. ADL, activities of daily living; AUC, area under the curve; IKDC, International Knee Documentation Committee; JR, joint replacement KOOS, Knee Injury and Osteoarthritis Outcome Score; MCID, minimal clinically important difference; PASS, patient-acceptable symptomatic state; QOL, quality of life; SCB, substantial clinical benefit. 
increase in their outcome scores. On the other hand, PASS represents postoperative scores rather than a change from baseline, which means patients with higher preoperative scores are already closer to achieving this threshold. The propensity for patients with greater symptoms and impairment to report improvement is particularly important and should be emphasized during clinical decision making and preoperative counseling.

Although all PROMs improved from 6 months to 1 year, this study found a relative decrease in the MCID thresholds for IKDC, Kujala (vs symptom), and KOOS Symptom, Pain, ADL, and Sport; a relative decrease in the SCB threshold for KOOS Symptom, ADL, QOL, and KOOS JR (vs. pain); and a relative decrease in the PASS threshold for KOOS Symptom, Kujala, and KOOS JR over the same time period. We also found that the MCID threshold for Kujala (vs symptom) at 6 months and KOOS JR (vs ADL) at 1 year were equivalent to the SCB threshold. Additionally, the SCB thresholds were found to be lower than those for MCID for KOOS Pain at 6 months, as well as KOOS Symptom, ADL, and QOL at 1 year. These discrepancies are likely attributable to a lower quantity of patient data available for analysis at 1 year, as well as for patients categorized into the "minimal change" group. One difficulty that arose in our calculations was the significant skewedness of patients failing into the "substantial improvement" category compared with the "no change" and "minimal improvement" categories, which may account for the relatively lower AUC values found for MCID. On the extreme end, zero patients fell into the "no change" groups for IKDC and Kujala (vs function) at 6 months, thus precluding any anchor-based analysis of those thresholds. Furthermore, we observed for many of the lowest AUC values that the average scores for the "no change" group were greater than the average values for the corresponding "minimal change" group, which is fairly intuitive because the ROC/AUC analysis evaluates how well the model distinguishes between the two groups and this inverse trend indicates a poor model. The relatively higher average values in these "no change" group were likely affected by the lower quantity of patient data available for analysis and may not reflect a true average. It remains to be seen if a larger sample size would help elucidate these trends, and further investigation is warranted.

\section{Limitations}

This study is not without inherent limitations. First, our study was underpowered because our patient follow-up at 1 year was $40 \%$. This limited our sample size and increased the risk of a selection bias in our cohort, as well as potentially reduced the generalizability and internal validity of these results. Second, while several commonly used PROMs related to knee symptoms, pain, and function were analyzed in this study, PROMs specific to patellar instability, such as the Banff Patella Instability Instrument and the Norwich Patellar Instability Score, were not included and, thus, limits the utility of these results for patients undergoing MPFL reconstruction. In our practice, these PROMs are not routinely administered, so these data were not available for analysis and additional inquiry into these PROMs and their outcome scores is needed. Third, the inclusion of minor concomitant procedures introduces a potential confounding variable. This effect was minimized by the exclusion of all major concomitant procedures that had the ability to alter surgical techniques or rehabilitation protocols. Given the relatively minor concomitant procedures included in this study, isolated and combination MPFL reconstructions were deemed to be sufficiently comparable for analysis. Fourth, the fact that $20.9 \%$ of our patient cohort had prior surgery on the index knee represents another potential confounding variable; however, the heterogeneity of these prior surgeries and the relatively low numbers of patients in each category precluded proper analysis of their effects. Further investigation with a larger patient population is needed to ascertain any influence that these variables and limitations may have. Lastly, this study was a retrospective review of prospectively collected data. As a result, we were unable to introduce an anchor question for anchor-based sensitivity analysis and, thus, were unable to perform any sensitivity analyses around our threshold values. The MCID, SCB, and PASS were calculated using a combination of anchor- and distribution-based methods, ${ }^{34,35}$ and neither is without its pitfalls nor do either demonstrate consistent superiority. Ideally, the disease under investigation and characteristics of the data should drive the determination of the methodologies used. ${ }^{34}$

\section{Conclusion}

This study established values for the MCID, SCB, and PASS at 6 months and 1 year after MPFLR, providing physicians an evidence-based method to advise patients and assess outcomes regarding this surgery. Older patients and those higher preoperative outcome scores are less likely to report improvement and satisfaction with MPFLR, while male patients are more likely to report some satisfaction.

\section{References}

1. Puzzitiello RN, Gowd AK, Liu JN, Agarwalla A, Verma NN, Forsythe B. Establishing minimal clinically important difference, substantial clinical benefit, and patient acceptable symptomatic state after biceps tenodesis. J Shoulder Elbow Surg 2019;28:639-647.

2. Nwachukwu BU, Chang B, Fields K, et al. Defining the "substantial clinical benefit" after arthroscopic treatment 
of femoroacetabular impingement. Am J Sports Med 2017;45:1297-1303.

3. Liu JN, Gowd AK, Redondo ML, et al. Establishing clinically significant outcomes after meniscal allograft transplantation. Orthop J Sports Med 2019;7: 2325967118818462.

4. Cvetanovich GL, Gowd AK, Liu JN, et al. Establishing clinically significant outcome after arthroscopic rotator cuff repair. J Shoulder Elbow Surg 2019;28:939-948.

5. Reagan J, Kullar R, Burks R. MPFL reconstruction: Technique and results. Orthop Clin North Am 2015;46: 159-169.

6. Mackay ND, Smith NA, Parsons N, Spalding T, Thompson P, Sprowson AP. Medial patellofemoral ligament reconstruction for patellar dislocation. Orthop J Sports Med 2014;2:2325967114544021.

7. Lee D, Park Y, Song S, Hwang S, Park J, Kang D. Which technique is better for treating patellar dislocation? A systematic review and meta-analysis. Arthroscopy 2018;34: 3082-3093.el.

8. Csintalan RP, Latt LD, Fornalski S, Raiszadeh K, Inacio MC, Fithian DC. Medial patellofemoral ligament (MPFL) reconstruction for the treatment of patellofemoral instability. J Knee Surg 2014;27:139-146.

9. Erickson BJ, Mascarenhas R, Sayegh ET, et al. Does operative treatment of first-time patellar dislocations lead to increased patellofemoral stability? A systematic review of overlapping meta-analyses. Arthroscopy 2015;31: 1207-1215.

10. Fisher B, Nyland J, Brand E, Curtin B. Medial patellofemoral ligament reconstruction for recurrent patellar dislocation: A systematic review including rehabilitation and return-to-sports efficacy. Arthroscopy 2010;26: 1384-1394.

11. Siebold R, Karidakis G, Fernandez F. Clinical outcome after medial patellofemoral ligament reconstruction and autologous chondrocyte implantation following recurrent patella dislocation. Knee Surg Sports Traumatol Arthrosc 2014;22:2477-2483.

12. Lyman S, Lee Y, Franklin P, Li W, Cross M, Padgett D. Validation of the KOOS, JR: A short-form knee arthroplasty outcomes survey. Clin Orthop Relat Res 2016;474: 1461-1471.

13. Kujala UM, Jaakkola LH, Koskinen SK, Taimela S, Hurme M, Nelimarkka O. Scoring of patellofemoral disorders. Arthroscopy 1993;9:159-163.

14. Roos EM, Roos HP, Lohmander LS, Ekdahl C, Beynnon BD. Knee injury and osteoarthritis outcome score (KOOS)-Development of a self-administered outcome measure. J Orthop Sports Phys Ther 1998;28: 88-96.

15. Irrgang JJ, Anderson AF, Boland AL, et al. Development and validation of the International Knee Documentation Committee subjective knee form. Am J Sports Med 2001;29:600-613.

16. King MT. A point of minimal important difference (MID): A critique of terminology and methods. Expert Rev Pharmacoeconomics Outcomes Res 2011;11:171-184.

17. Tubach F, Ravaud P, Baron G, et al. Evaluation of clinically relevant states in patient reported outcomes in knee and hip osteoarthritis: The patient acceptable symptom state. Ann Rheum Dis 2005;64:34-37.

18. Anbari A, Cole BJ. Medial patellofemoral ligament reconstruction-A novel approach. J Knee Surg 2008;21: 241-245.

19. Patel NK, de SA D, Vaswani R, Kay J, Musahl V, Lesniak BP. Knee flexion angle during graft fixation for medial patellofemoral ligament reconstruction: A systematic review of outcomes and complications. Arthroscopy 2019;35:1893-1904.

20. Weinberger JM, Fabricant PD, Taylor SA, Mei JY, Jones KJ. Influence of graft source and configuration on revision rate and patient-reported outcomes after MPFL reconstruction: A systematic review and meta-analysis. Knee Surg Sports Traumatol Arthrosc 2016;25:2511-2519.

21. Norman GR, Sloan JA, Wyrwich KW. Interpretation of changes in health-related quality of life: The remarkable universality of half a standard deviation. Med Care 2003;41:582-592.

22. Chahal J, Van Thiel GS, Mather RC, et al. The patient acceptable symptomatic state for the modified Harris hip score and hip outcome score among patients undergoing surgical treatment for femoroacetabular impingement. Am J Sports Med 2015;43:1844-1849.

23. Kukkonen J, Kauko T, Vahlberg T, Joukainen A, Äärimaa V. Investigating minimal clinically important difference for Constant score in patients undergoing rotator cuff surgery. J Shoulder Elbow Surg 2013;22: $1650-1655$.

24. Tashjian R, Deloach J, Green A, Porucznik C, Powell A. Minimal clinically important differences in ASES and simple shoulder test scores after nonoperative treatment of rotator cuff disease. J Bone Joint Surg Am 2010;92: 296-303.

25. U.S. Department of Health and Human Services FDA Center for Drug Evaluation and Research; U.S. Department of Health and Human Services FDA Center for Biologics Evaluation and Research; U.S. Department of Health and Human Services FDA Center for Devices and Radiological Health. Guidance for industry: Patientreported outcome measures: Use in medical product development to support labeling claims: Draft guidance. Health Qual Life Outcomes 2006;4:79.

26. Ward MM, Guthrie LC, Alba M. Domain-specific transition questions demonstrated higher validity than global transition questions as anchors for clinically important improvement. J Clinic Epidemiol 2015;68:655-661.

27. Crosby RD, Kolotkin RL, Williams GR. Defining clinically meaningful change in health-related quality of life. J Clinic Epidemiol 2003;56:395-407.

28. Schneider DK, Grawe B, Magnussen RA, et al. Outcomes after isolated medial patellofemoral ligament reconstruction for the treatment of recurrent lateral patellar dislocations. Am J Sports Med 2016;44:2993-3005.

29. Sappey-Marinier E, Sonnery-Cottet B, O'Loughlin P, et al. Clinical outcomes and predictive factors for failure with isolated MPFL reconstruction for recurrent patellar instability: A series of 211 reconstructions with a minimum follow-up of 3 years. Am J Sports Med 2019;47: 1323-1330. 
30. Hiemstra LA, Kerslake S. Age at time of surgery but not sex is related to outcomes after medial patellofemoral ligament reconstruction. Am J Sports Med 2019;47: 1638-1644.

31. Nwachukwu BU, Fields K, Chang B, Nawabi DH, Kelly BT, Ranawat AS. Preoperative outcome scores are predictive of achieving the minimal clinically important difference after arthroscopic treatment of femoroacetabular impingement. Am J Sports Med 2017;45:612-619.

32. Gowd AK, Lalehzarian SP, Liu JN, et al. Factors associated with clinically significant patient-reported outcomes after primary arthroscopic partial meniscectomy. Arthroscopy 2019;35:1567-1575.e3.
33. Cvetanovich GL, Weber AE, Kuhns BD, et al. Hip arthroscopic surgery for femoroacetabular impingement with capsular management: Factors associated with achieving clinically significant outcomes. Am J Sports Med 2018;46:288-296.

34. Nwachukwu BU, Chang B, Voleti PB, et al. Preoperative short form health survey score is predictive of return to play and minimal clinically important difference at a minimum 2-year follow-up after anterior cruciate ligament reconstruction. Am J Sports Med 2017;45:2784-2790.

35. Jaeschke R, Singer J, Guyatt GH. Measurement of health status: Ascertaining the minimal clinically important difference. Control Clin Trials 1989;10:407-415. 


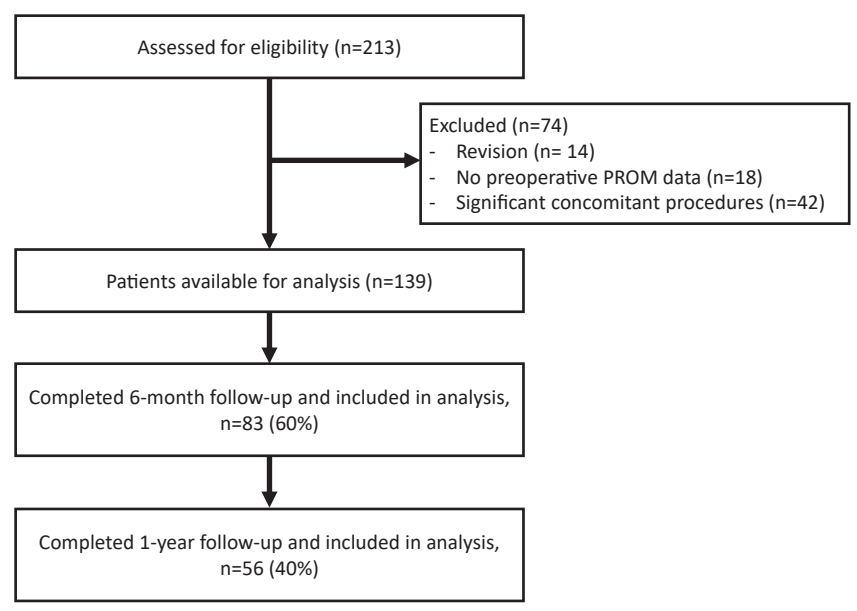

Appendix Fig. 1. STROBE Flow Diagram of Development of Cohort

Appendix Table 1. Net Change in Outcome Scores at 6 Months and 1 Year Postoperatively

\begin{tabular}{lcc}
\hline \multirow{2}{*}{\multicolumn{1}{c}{ Assessment }} & \multicolumn{2}{c}{ Change in Outcome Scores } \\
\cline { 2 - 3 } & 6 Months & 1 Year \\
\hline IKDC & $25.50 \pm 19.36$ & $30.92 \pm 17.17$ \\
KOOS Symptom & $17.67 \pm 19.28$ & $17.75 \pm 21.70$ \\
KOOS Pain & $20.06 \pm 20.63$ & $20.52 \pm 19.84$ \\
KOOS ADL & $18.38 \pm 18.73$ & $18.64 \pm 21.33$ \\
KOOS Sport & $34.50 \pm 24.89$ & $35.47 \pm 28.09$ \\
KOOS QOL & $30.92 \pm 24.44$ & $41.48 \pm 24.79$ \\
Kujala vs Function & $27.08 \pm 16.92$ & $30.10 \pm 19.87$ \\
Kujala vs Symptom & $25.86 \pm 18.23$ & $29.97 \pm 19.57$ \\
KOOS JR vs Pain & $14.54 \pm 14.92$ & $20.87 \pm 15.87$ \\
KOOS JR vs Symptom & $13.49 \pm 15.25$ & $20.34 \pm 16.17$ \\
KOOS JR vs ADL & $13.95 \pm 15.47$ & $20.34 \pm 16.17$ \\
\hline
\end{tabular}

IKDC, International Knee Documentation Committee; KOOS, Knee injury and Osteoarthritis Outcome Score; ADL, Activities of Daily Living; QOL, Quality of Life; JR, Joint Replacement. 


\begin{tabular}{|c|c|c|c|c|c|c|c|}
\hline \multirow[b]{2}{*}{ Questionnaire } & \multirow[b]{2}{*}{ Factor } & \multicolumn{3}{|c|}{6 Months } & \multicolumn{3}{|c|}{12 Months } \\
\hline & & Univariate & Multivariate & OR $(95 \%$ CI $)$ & Univariate & Multivariate & OR $(95 \%$ CI $)$ \\
\hline \multirow[t]{7}{*}{ IKDC } & Sex & 0.850 & & $1.111(0.372-3.320)$ & 0.860 & & $0.897(0.269-2.988)$ \\
\hline & Age & 0.004 & 0.001 & $0.861(0.789-0.939)$ & 0.446 & & $0.975(0.914-1.040)$ \\
\hline & BMI & 0.174 & & $0.950(0.883-1.022)$ & 0.477 & & $0.972(0.898-1.052)$ \\
\hline & WC & 0.134 & & $0.148(0.013-1.722)$ & 0.930 & & $1.118(0.095-13.150)$ \\
\hline & Age at First Instability Event & 0.137 & & $0.972(0.908-1.040)$ & 0.562 & & $1.023(0.948-1.103)$ \\
\hline & Number of Instability Events & 0.483 & & $0.989(0.958-1.020)$ & 0.253 & & $0.954(0.880-1.034)$ \\
\hline & Preoperative PRO Score & 0.027 & 0.004 & $0.946(0.911-0.983)$ & 0.010 & & $0.954(0.921-0.989)$ \\
\hline \multirow[t]{7}{*}{ KOOS Symptoms } & Sex & 0.313 & & $0.478(0.114-2.004)$ & 0.509 & & $1.625(0.384-6.872)$ \\
\hline & Age & 0.803 & & $1.010(0.933-1.094)$ & 0.342 & & $1.049(0.950-1.159)$ \\
\hline & BMI & 0.093 & .901 & $1.009(0.877-1.160)$ & 0.260 & & $1.061(0.957-1.177)$ \\
\hline & WC & 0.685 & & $0.556(0.032-9.535)$ & 0.878 & & $0.800(0.046-13.812)$ \\
\hline & Age at First Instability Event & 0.828 & & $0.992(0.919-1.070)$ & 0.158 & & $1.087(0.968-1.219)$ \\
\hline & Number of Instability Events & 0.261 & & $1.160(0.895-1.503)$ & 0.397 & & $0.944(0.826-1.079)$ \\
\hline & Preoperative PRO Score & 0.001 & 0.002 & $0.911(0.857-0.967)$ & 0.004 & & $0.906(0.847-0.968)$ \\
\hline \multirow[t]{7}{*}{ KOOS Pain } & Sex & 0.234 & & $0.417(0.098-1.763)$ & 0.740 & & $0.762(0.153-3.802)$ \\
\hline & Age & 0.600 & & $1.022(0.942-1.110)$ & 0.484 & & $1.043(0.926-1.176)$ \\
\hline & BMI & 0.137 & & $1.091(0.973-1.122)$ & 0.289 & & $1.081(0.936-1.247)$ \\
\hline & WC & 0.633 & & $0.726(0.029-8.602)$ & predicts success perfectly & & \\
\hline & Age at First Instability Event & 0.105 & & $1.089(0.982-1.208)$ & 0.078 & 0.176 & $1.1456(0.941-1.340)$ \\
\hline & Number of Instability Events & 0.355 & & $0.964(0.891-1.042)$ & 0.170 & & $0.791(0.565-1.106)$ \\
\hline & Preoperative PRO Score & 0.032 & & $0.961(0.926-0.996)$ & 0.036 & 0.100 & $0.949(0.890-1.010)$ \\
\hline \multirow[t]{7}{*}{ KOOS ADL } & Sex & 0.229 & & $0.413(0.098-1.745)$ & 0.885 & & $1.123(0.235-5.359)$ \\
\hline & Age & 0.910 & & $1.004(0.930-1.085)$ & 0.426 & & $1.046(0.936-1.170)$ \\
\hline & BMI & 0.212 & & $1.064(0.965-1.173)$ & 0.321 & & $1.065(0.941-1.204)$ \\
\hline & WC & 0.843 & & $0.750(0.044-12.816)$ & 0.513 & & $0.385(0.022-6.757)$ \\
\hline & Age at First Instability Event & 0.284 & & $1.046(0.964-1.134)$ & 0.065 & 0.205 & $1.157(0.923-1.451)$ \\
\hline & Number of Instability Events & 0.299 & & $0.950(0.863-1.047)$ & 0.298 & & $0.917(0.780-1.079)$ \\
\hline & Preoperative PRO Score & 0.001 & & $0.878(0.814-0.948)$ & 0.007 & 0.032 & $0.912(0.839-0.992)$ \\
\hline \multirow[t]{7}{*}{ KOOS Sport } & Sex & 0.456 & & $2.333(0.252-21.634)$ & 0.355 & & $0.505(0.119-2.145)$ \\
\hline & Age & 0.539 & & $0.971(0.882-1.068)$ & 0.919 & & $1.005(0.909-1.112)$ \\
\hline & BMI & 0.275 & & $1.086(0.936-1.261)$ & 0.568 & & $0.973(0.884-1.070)$ \\
\hline & WC & 0.275 & & $0.200(0.011-3.592)$ & 0.634 & & $0.500(0.029-8.706)$ \\
\hline & Age at First Instability Event & 0.949 & & $0.997(0.906-1.096)$ & 0.450 & & $1.044(0.934-1.167)$ \\
\hline & Number of Instability Events & 0.692 & & $1.027(0.901-1.170)$ & 0.304 & & $0.931(0.811-1.067)$ \\
\hline & Preoperative PRO Score & 0.096 & & $0.974(0.944-1.005)$ & 0.092 & & $0.976(0.948-1.004)$ \\
\hline \multirow[t]{7}{*}{ KOOS QOL } & Sex & 0.679 & & $0.718(0.150-3.442)$ & predicts success perfectly & & Collinearity \\
\hline & Age & 0.731 & & $0.985(0.904-1.074)$ & 0.712 & & $1.032(0.875-1.216)$ \\
\hline & BMI & 0.390 & & $1.052(0.937-1.180)$ & 0.854 & & $1.015(0.865-1.191)$ \\
\hline & WC & 0.426 & & $0.313(0.179-5.464)$ & predicts success perfectly & & \\
\hline & Age at First Instability Event & 0.421 & & $1.040(0.945-1.145)$ & 0.444 & & $1.074(0.895-1.289)$ \\
\hline & Number of Instability Events & 0.171 & & $1.310(0.890-1.929)$ & 0.975 & & $1.002(0.859-1.170)$ \\
\hline & Preoperative PRO Score & 0.084 & & $0.970(0.936-1.004)$ & 0.522 & & $1.025(0.951-1.105)$ \\
\hline
\end{tabular}




\begin{tabular}{|c|c|c|c|c|c|c|c|}
\hline \multirow[b]{2}{*}{ Questionnaire } & \multirow[b]{2}{*}{ Factor } & \multicolumn{3}{|c|}{6 Months } & \multicolumn{3}{|c|}{12 Months } \\
\hline & & Univariate & Multivariate & OR $(95 \%$ CI $)$ & Univariate & Multivariate & OR $(95 \% \mathrm{CI})$ \\
\hline \multirow[t]{7}{*}{ Kujala Function } & Sex & 0.387 & & $0.500(0.104-2.403)$ & predicts success perfectly & & Collinearity \\
\hline & Age & 0.755 & & $0.981(0.890-1.088)$ & predicts success perfectly & & \\
\hline & BMI & 0.699 & & $0.980(0.886-1.085)$ & predicts success perfectly & & \\
\hline & WC & 0.324 & & $0.280(0.022-3.506)$ & predicts success perfectly & & \\
\hline & Age at First Instability Event & 0.551 & & $0.969(0.873-1.075)$ & predicts success perfectly & & \\
\hline & Number of Instability Events & 0.931 & & $1.002(0.950-1.058)$ & 0.369 & & $0.741(0.386-1.424)$ \\
\hline & Preoperative PRO Score & 0.088 & & $0.959(0.913-1.006)$ & predicts success perfectly & & \\
\hline \multirow[t]{7}{*}{ Kujala Symptoms } & Sex & 0.856 & & $1.143(0.269-4.859)$ & predicts success perfectly & & Collinearity \\
\hline & Age & 0.051 & 0.013 & $0.862(0.766-0.970)$ & predicts success perfectly & & \\
\hline & BMI & 0.298 & & $0.957(0.881-1.040)$ & predicts success perfectly & & \\
\hline & WC & 0.094 & 0.234 & $0.182(0.011-3.000)$ & predicts success perfectly & & \\
\hline & Age at First Instability Event & 0.214 & & $0.947(0.870-1.032)$ & predicts success perfectly & & \\
\hline & Number of Instability Events & 0.188 & & $0.976(0.942-1.012)$ & 0.369 & & $0.741(0.386-1.424)$ \\
\hline & Preoperative PRO Score & 0.043 & 0.007 & $0.931(0.884-0.980)$ & predicts success perfectly & & \\
\hline \multirow[t]{7}{*}{ KOOS JR Pain } & Sex & 0.894 & & $1.071(0.387-2.968)$ & 0.932 & & $1.067(0.241-4.715)$ \\
\hline & Age & 0.792 & & $1.008(0.947-1.074)$ & 0.968 & & $1.002(0.903-1.112)$ \\
\hline & BMI & 0.204 & & $1.051(0.973-1.135)$ & 0.591 & & $1.031(0.923-1.152)$ \\
\hline & WC & 0.925 & & $1.125(0.097-12.989)$ & predicts success perfectly & & \\
\hline & Age at First Instability Event & 0.659 & & $1.015(0.950-1.085)$ & 0.099 & 0.830 & $1.023(0.830-1.260)$ \\
\hline & Number of Instability Events & 0.431 & & $1.017(0.975-1.060)$ & 0.098 & 0.130 & $0.922(0.830-1.024)$ \\
\hline & Preoperative PRO Score & 0.066 & & $0.969(0.937-1.002)$ & 0.087 & 0.020 & $0.907(0.835-0.985)$ \\
\hline \multirow[t]{7}{*}{ KOOS JR Symptoms } & Sex & 0.894 & & $1.071(0.387-2.968)$ & 0.631 & & $1.429(0.333-6.131)$ \\
\hline & Age & 0.972 & & $1.009(0.947-1.074)$ & 0.649 & & $1.024(0.926-1.132)$ \\
\hline & BMI & 0.204 & & $1.051(0.973-1.135)$ & 0.381 & & $1.050(0.941-1.172)$ \\
\hline & WC & 0.925 & & $1.125(0.097-12.989)$ & predicts success perfectly & & \\
\hline & Age at First Instability Event & 0.659 & & $1.015(0.950-1.085)$ & 0.109 & & $1.138(0.972-1.333)$ \\
\hline & Number of Instability Events & 0.431 & & $1.017(0.975-1.060)$ & 0.156 & & $0.944(0.871-1.022)$ \\
\hline & Preoperative PRO Score & 0.066 & & $0.969(0.937-1.002)$ & 0.003 & & $0.899(0.839-0.964)$ \\
\hline \multirow[t]{7}{*}{ KOOS JR ADL } & Sex & 0.894 & & $1.071(0.387-2.968)$ & 0.631 & & $1.429(0.333-6.131)$ \\
\hline & Age & 0.792 & & $1.009(0.947-1.074)$ & 0.649 & & $1.024(0.926-1.132)$ \\
\hline & BMI & 0.204 & & $1.051(0.973-1.135)$ & 0.381 & & $1.050(0.941-1.172)$ \\
\hline & WC & 0.925 & & $1.125(0.097-12.989)$ & predicts success perfectly & & \\
\hline & Age at First Instability Event & 0.659 & & $1.015(0.950-1.085)$ & 0.109 & & $1.138(0.971-1.333)$ \\
\hline & Number of Instability Events & 0.431 & & $1.017(0.975-1.060)$ & 0.156 & & $0.944(0.871-1.022)$ \\
\hline & Preoperative PRO Score & 0.066 & & $0.969(0.937-1.002)$ & 0.003 & & $0.899(0.837-0.964)$ \\
\hline
\end{tabular}

and OR does not Cross 1.0$)$ All univarite and mulivaniate analys Documentation Committee; KOOS, Knee Injury and Osteoarthritis Outcome Score; ADL, activities of daily living; QOL, quality of life; JR, joint replacement; BMI, body mass index; WC, workers' compensation; PRO, patient-reported outcome. 
Appendix Table 3. Logistic Regression of Variables Associated with Achieving SCB at 6 Months and 1 Year

\begin{tabular}{|c|c|c|c|c|c|c|c|}
\hline \multirow[b]{2}{*}{ Questionnaire } & \multirow[b]{2}{*}{ Factor } & \multicolumn{3}{|c|}{6 Months } & \multicolumn{3}{|c|}{12 Months } \\
\hline & & Univariate & Multivariate & OR $(95 \% \mathrm{CI})$ & Univariate & Multivariate & OR $(95 \% \mathrm{CI})$ \\
\hline \multirow[t]{7}{*}{$\mathrm{IKDC}$} & Sex & & & & .860 & & $.897(.270-2.988)$ \\
\hline & Age & & & & .446 & & $.975(.914-1.040)$ \\
\hline & BMI & & & & .477 & & $.972(.898-1.052)$ \\
\hline & WC & & & & .930 & & $1.118(.095-13.150)$ \\
\hline & Age at First Instability Event & & & & .562 & & $1.023(.948-1.103)$ \\
\hline & Number of Instability Events & & & & .253 & & $.954(.880-1.034)$ \\
\hline & Preoperative PRO Score & & & & .010 & & $.954(.921-.989)$ \\
\hline \multirow[t]{7}{*}{ KOOS Symptoms } & Sex & 1.000 & & $1.000(.244-4.097)$ & predicts success perfectly & & \\
\hline & Age & .536 & & $.976(.904-1.054)$ & .845 & & $1.011(.903-1.133)$ \\
\hline & BMI & .298 & & $1.050(.958-1.150)$ & .313 & & $1.075(.934-1.237)$ \\
\hline & WC & 1.000 & & $1.000(.059-17.065)$ & predicts success perfectly & & \\
\hline & Age at First Instability Event & .652 & & $.983(.912-1.059)$ & .970 & & $.998(.887-1.122)$ \\
\hline & Number of Instability Events & .392 & & $1.041(.949-1.142)$ & .838 & & $1.012(.902-1.135)$ \\
\hline & Preoperative PRO Score & .002 & & $.930(.888-.975)$ & .010 & & $.856(.761-.963)$ \\
\hline \multirow[t]{7}{*}{ KOOS Pain } & Sex & .047 & .333 & $.435(.081-2.347)$ & .438 & & $.545(.118-2.526)$ \\
\hline & Age & .764 & & $1.014(.927-1.109)$ & .072 & .801 & $1.025(.845-1.244)$ \\
\hline & BMI & .242 & & $1.078(.951-1.222)$ & .045 & .692 & $1.032(.883-1.206)$ \\
\hline & WC & predicts success perfectly & & & .695 & & $1.769(.102-30.709)$ \\
\hline & Age at First Instability Event & .247 & & $1.064(.958-1.181)$ & .057 & .732 & $1.039(.836-1.291)$ \\
\hline & Number of Instability Events & .251 & & $.955(.883-1.033)$ & .708 & & $.982(.890-1.082)$ \\
\hline & Preoperative PRO Score & .021 & .072 & $.958(.914-1.004)$ & .003 & .009 & $.892(.819-.972)$ \\
\hline \multirow[t]{7}{*}{ KOOS ADL } & Sex & .191 & & $.232(.026-2.070)$ & .885 & & $1.123(.235-5.359)$ \\
\hline & Age & .791 & & $.988(.906-1.078)$ & .426 & & $1.046(.936-1.170)$ \\
\hline & BMI & .069 & .469 & $1.042(.932-1.166)$ & .321 & & $1.065(.941-1.204)$ \\
\hline & WC & .477 & & $2.818(.162-49.008)$ & .513 & & $.385(.022-6.757)$ \\
\hline & Age at first instability event & .591 & & $1.022(.943-1.108)$ & .065 & .409 & $.958(.866-1.060)$ \\
\hline & Number of instability events & .622 & & $.973(.874-1.084)$ & .298 & & $.917(.780-1.079)$ \\
\hline & Preoperative PRO score & .003 & .007 & $.937(.894-.983)$ & .007 & .004 & $.924(.876-.975)$ \\
\hline \multirow[t]{7}{*}{ KOOS Sport } & Sex & .295 & & $.309(.034-2.791)$ & .716 & & $.750(.159-3.532)$ \\
\hline & Age & .692 & & $.981(.894-1.077)$ & .371 & & $1.048(.946-1.161)$ \\
\hline & BMI & .638 & & $1.024(.928-1.130)$ & 0.267 & & $1.057(.959-1.164)$ \\
\hline & WC & predicts success perfectly & & & predicts success perfectly & & \\
\hline & Age at first instability event & .591 & & $1.024(.940-1.114)$ & 0.475 & & $1.038(.937-1.151)$ \\
\hline & Number of instability events & .670 & & $.975(.870-1.094)$ & .771 & & $.985(.892-1.089)$ \\
\hline & Preoperative PRO score & .035 & & $.956(.917-.997)$ & .013 & & $.944(.901-.988)$ \\
\hline
\end{tabular}




\begin{tabular}{|c|c|c|c|c|c|c|c|}
\hline \multirow[b]{2}{*}{ Questionnaire } & \multirow[b]{2}{*}{ Factor } & \multicolumn{3}{|c|}{6 Months } & \multicolumn{3}{|c|}{12 Months } \\
\hline & & Univariate & Multivariate & OR $(95 \% \mathrm{CI})$ & Univariate & Multivariate & OR $(95 \% \mathrm{CI})$ \\
\hline \multirow[t]{7}{*}{ KOOS QOL } & Sex & .888 & & $.896(.194-4.145)$ & predicts success perfectly & & collinearity \\
\hline & Age & .322 & & $.957(.876-1.044)$ & .712 & & $1.032(.875-1.216)$ \\
\hline & BMI & .712 & & $.982(.892-1.080)$ & .854 & & $1.015(.865-1.190)$ \\
\hline & WC & predicts success perfectly & & & predicts success perfectly & & \\
\hline & Age at First Instability Event & .164 & & $.931(.844-1.029)$ & .444 & & $1.074(.895-1.289)$ \\
\hline & Number of Instability Events & .412 & & $1.032(.957-1.112)$ & .975 & & $1.002(.859-1.170)$ \\
\hline & Preoperative PRO Score & .051 & & $.952(.906-1.000)$ & .522 & & $1.025(.951-1.105)$ \\
\hline \multirow[t]{7}{*}{ Kujala Function } & Sex & & & & predicts success perfectly & & collinearity \\
\hline & Age & & & & .186 & & $.859(.686-1.076)$ \\
\hline & BMI & & & & .242 & & $.844(.636-1.121)$ \\
\hline & WC & & & & .858 & & $.750(.032-17.506)$ \\
\hline & Age at first instability event & & & & .165 & & $.833(.643-1.078)$ \\
\hline & Number of instability events & & & & .753 & & $1.089(.639-1.856)$ \\
\hline & Preoperative PRO score & & & & .296 & & $1.04(.967-1.118)$ \\
\hline \multirow[t]{7}{*}{ Kujala Symptoms } & Sex & .856 & & $1.143(.269-4.859)$ & predicts success perfectly & & \\
\hline & Age & .051 & .013 & $.862(.766-.970)$ & .186 & & $.859(.686-1.076)$ \\
\hline & BMI & .298 & & $.957(.881-1.040)$ & .242 & & $1.076(.636-1.121)$ \\
\hline & WC & .094 & .234 & $.182(.011-3.000)$ & .858 & & $.75(.032-17.506)$ \\
\hline & Age at first instability event & .214 & & $.947(.870-1.032)$ & .165 & & $.833(.643-1.078)$ \\
\hline & Number of instability events & .188 & & $.976(.942-1.012)$ & .753 & & $1.089(.639-1.856)$ \\
\hline & Preoperative PRO score & .043 & .007 & $.931(.884-.980)$ & .296 & & $1.04(.967-1.118)$ \\
\hline \multirow[t]{7}{*}{ KOOS JR Pain } & Sex & .110 & & $.335(.088-1.282)$ & .356 & & $.556(.159-1.935)$ \\
\hline & Age & .243 & & $.956(.887-1.031)$ & .582 & & $1.024(.941-1.115)$ \\
\hline & BMI & .564 & & $1.022(.948-1.102)$ & .529 & & $1.027(.945-1.117)$ \\
\hline & WC & .757 & & $1.474(.126-17.184)$ & .848 & & $1.318(.078-22.263)$ \\
\hline & Age at First Instability Event & .724 & & $.987(0.916-1.062)$ & .300 & & $1.054(.954-1.164)$ \\
\hline & Number of Instability Events & .529 & & $.985(.941-1.032)$ & .862 & & $.994(.926-1.067)$ \\
\hline & Preoperative PRO score & .001 & & $.912(.864-.964)$ & .008 & & $.930(.881-.981)$ \\
\hline \multirow[t]{7}{*}{ KOOS JR Symptoms } & Sex & .110 & & $.335(.088-1.282)$ & .726 & & $.788(.208-2.989)$ \\
\hline & Age & .243 & & $.956(.887-1.031)$ & .952 & & $1.003(.915-1.099)$ \\
\hline & BMI & .564 & & $1.022(.948-1.102)$ & .195 & & $1.061(.970-1.159)$ \\
\hline & WC & .757 & & $1.474(.126-17.184)$ & .545 & & $2.400(.141-40.934)$ \\
\hline & Age at first instability event & .724 & & $.987(.916-1.062)$ & .191 & & $1.071(.966-1.188)$ \\
\hline & Number of instability events & .529 & & $.985(.941-1.032)$ & .476 & & $.961(.860-1.073)$ \\
\hline & Preoperative PRO score & .001 & & $.912(.864-.964)$ & .002 & & $.822(.728-.928)$ \\
\hline \multirow[t]{7}{*}{ KOOS JR ADL } & Sex & .235 & & $.439(.113-1.706)$ & .384 & & $.558(.149-2.080)$ \\
\hline & Age & .385 & & $.966(.893-1.044)$ & .733 & & $1.015(.930-1.108)$ \\
\hline & BMI & .262 & & $1.045(.967-1.129)$ & .297 & & $1.047(.960-1.141)$ \\
\hline & WC & .626 & & $1.844(.157-21.650)$ & .675 & & $1.833(.108-31.088)$ \\
\hline & Age at first instability event & .721 & & $.986(.911-1.067)$ & .223 & & $1.065(.962-1.178)$ \\
\hline & Number of instability events & .583 & & $.986(.940-1.036)$ & .994 & & $1.000(.931-1.074)$ \\
\hline & Preoperative PRO score & .005 & & $.929(.883-.978)$ & .002 & & $.892(.830-.958)$ \\
\hline
\end{tabular}

Bold indicates significant value $(P<.05$ and OR does not cross 1.0). All univariate and multivariate analyses reported as $P$ values. The odds ratio is reported for multivariate regression if significant, otherwise corresponds to univariate regression. OR, odds ratio; 95\% CI, 95\% confidence interval; SCB, substantial clinical benefit; IKDC, International Knee Documentation Committee; KOOS, Knee Injury and Osteoarthritis Outcome Score; ADL, activities of daily living; QOL, quality of life; JR, joint replacement; BMI, body mass index; WC, workers' compensation; PRO, patient-reported outcome. 
Appendix Table 4. Logistic Regression of Variables Associated With Achieving PASS at 6 Months and 1 Year

\begin{tabular}{|c|c|c|c|c|c|c|c|}
\hline \multirow[b]{2}{*}{ Questionnaire } & \multirow[b]{2}{*}{ Factor } & \multicolumn{3}{|c|}{6 Months } & \multicolumn{3}{|c|}{12 Months } \\
\hline & & Univariate & Multivariate & Or $(95 \% \mathrm{CI})$ & Univariate & Multivariate & Or $(95 \% \mathrm{CI})$ \\
\hline \multirow[t]{7}{*}{ IKDC } & Sex & .028 & .073 & $2.950(.905-9.623)$ & .884 & & $1.098(.313-3.847)$ \\
\hline & Age & .004 & .348 & $.963(.891-1.041)$ & .014 & .983 & $.999(.899-1.110)$ \\
\hline & BMI & .004 & .262 & $.952(.874-1.037)$ & .001 & .020 & $.885(.799-.981)$ \\
\hline & WC & .609 & & $.643(.118-3.496)$ & .078 & .585 & $.530(.055-5.150)$ \\
\hline & Age at first instability event & .012 & .846 & $.992(.916-1.074)$ & .013 & .849 & $.990(.889-1.102)$ \\
\hline & Number of instability events & .439 & & $.990(.965-1.016)$ & .637 & & $1.017(.948-1.090)$ \\
\hline & Preoperative PRO score & .002 & .082 & $1.028(.996-1.060)$ & .033 & .344 & $1.022(.977-1.070)$ \\
\hline \multirow[t]{7}{*}{ KOOS Symptoms } & Sex & .093 & .432 & $1.587(.502-5.020)$ & .117 & & $2.825(.770-10.360)$ \\
\hline & Age & .054 & .016 & $.900(.825-0.980)$ & .936 & & $.997(.938-1.060)$ \\
\hline & BMI & .015 & .854 & $.990(.892-1.099)$ & .175 & & $.948(.877-1.024)$ \\
\hline & WC & .694 & & $1.543(.178-13.363)$ & .865 & & $.828(.093-7.325)$ \\
\hline & Age at first instability Event & .114 & & $.962(.917-1.009)$ & .793 & & $1.009(.943-1.080)$ \\
\hline & Number of instability events & .190 & & $1.070(.967-1.185)$ & .872 & & $.997(.963-1.032)$ \\
\hline & Preoperative PRO score & .078 & .419 & $1.013(.982-1.044)$ & .238 & & $1.018(.988-1.049)$ \\
\hline \multirow[t]{7}{*}{ KOOS Pain } & Sex & .073 & .166 & $8.470(.412-174.335)$ & .059 & & $7.385(.930-58.606)$ \\
\hline & Age & .002 & .022 & $.823(.696-.973)$ & .367 & & $.972(.914-1.034)$ \\
\hline & BMI & .003 & .778 & $1.024(.869-1.206)$ & .597 & & $.976(.891-1.069)$ \\
\hline & WC & .590 & & $.545(.061-4.948)$ & .649 & & $.600(.067-5.407)$ \\
\hline & Age at first instability event & .009 & .493 & $.950(.821-1.100$ & .936 & & $.997(.929-1.070)$ \\
\hline & Number of instability events & .450 & & $1.047(.930-1.178)$ & .796 & & $.995(.959-1.032)$ \\
\hline & Preoperative PRO score & .004 & .059 & $1.060(.998-1.127)$ & .368 & & $1.015(.983-1.048)$ \\
\hline \multirow[t]{7}{*}{ KOOS ADL } & Sex & .068 & .424 & $1.923(.388-9.536)$ & .033 & & $3.424(1.102-10.634)$ \\
\hline & Age & .020 & .040 & $.878(.776-.994)$ & .918 & & $.997(.947-1.051)$ \\
\hline & BMI & .013 & .605 & $1.035(.908-1.179)$ & .822 & & $.992(.922-1.066)$ \\
\hline & WC & .961 & & $.947(.108-8.335)$ & .461 & & $.527(.096-2.889)$ \\
\hline & Age at first instability event & .040 & .593 & $.970(.869-1.084)$ & .764 & & $1.009(.953-1.068)$ \\
\hline & Number of instability events & .324 & & $1.056(.948-1.176)$ & .841 & & $1.003(.970-1.038)$ \\
\hline & Preoperative PRO score & .005 & .074 & $1.035(.997-1.074)$ & .156 & & $1.018(.993-1.048)$ \\
\hline \multirow[t]{7}{*}{ KOOS Sport } & Sex & .176 & & $2.938(.617-11.983$ & .719 & & $1.293(.319-5.242)$ \\
\hline & Age & .019 & .399 & $.941(.817-1.084)$ & .449 & & $.973(.908-1.043)$ \\
\hline & BMI & .003 & .644 & $.969(.848-1.108)$ & .036 & .221 & $.937(.844-1.040)$ \\
\hline & WC & .590 & & $.545(.060-4.948)$ & .468 & & $.439(.048-4.053)$ \\
\hline & Age at first instability event & .025 & .634 & $.970(.855-1.100)$ & .986 & & $1.001(.922-1.086)$ \\
\hline & Number of instability events & .411 & & $1.060(.922-1.219)$ & .585 & & $.990(.955-1.026)$ \\
\hline & Preoperative PRO score & .005 & .053 & $1.047(.999-1.096)$ & .062 & .164 & $1.026(.989-1.064)$ \\
\hline \multirow[t]{7}{*}{ KOOS QOL } & Sex & .341 & & $1.917(.502-7.321)$ & .307 & & $2.293(.467-11.248)$ \\
\hline & Age & .105 & & $0.953(.898-1.010)$ & .372 & & $.969(.905-1.038)$ \\
\hline & BMI & .052 & .209 & $.942(.858-1.034)$ & .630 & & $.975(.881-1.080)$ \\
\hline & WC & .649 & & $.600(.067-5.407)$ & .468 & & $.439(.04-4.053)$ \\
\hline & Age at first instability event & .207 & & $0.962(.907-1.021)$ & .943 & & $1.003(.923-1.090)$ \\
\hline & Number of instability events & .433 & & $1.05(.934-1.171)$ & .601 & & $.990(.955-1.027)$ \\
\hline & Preoperative PRO score & .027 & .045 & $1.053(1.001-1.108)$ & .098 & & $1.043(.992-1.098)$ \\
\hline
\end{tabular}




\begin{tabular}{|c|c|c|c|c|c|c|c|}
\hline \multirow[b]{2}{*}{ Questionnaire } & \multirow[b]{2}{*}{ Factor } & \multicolumn{3}{|c|}{6 Months } & \multicolumn{3}{|c|}{12 Months } \\
\hline & & Univariate & Multivariate & Or $(95 \% \mathrm{CI})$ & Univariate & Multivariate & Or $(95 \% \mathrm{CI})$ \\
\hline \multirow[t]{7}{*}{ KOOS JR } & Sex & .179 & & $1.761(.771-4.022)$ & .134 & & $3.272(.695-15.406)$ \\
\hline & Age & .010 & .065 & $.926(0.853-1.005)$ & .102 & & $.952(.898-1.001)$ \\
\hline & BMI & .019 & .955 & $1.002(.923-1.089)$ & .292 & & $.954(.875-1.041)$ \\
\hline & WC & .354 & & $.481(.102-2.258)$ & .649 & & $.600(.067-5.407)$ \\
\hline & Age at first instability event & .024 & .862 & $.993(.918-1.075)$ & .854 & & $.993(.927-1.065)$ \\
\hline & Number of Instability Events & .927 & & $.999(.973-1.026)$ & .542 & & $.990(.958-1.023)$ \\
\hline & Preoperative PRO Score & .006 & .059 & $1.032(.999-1.067)$ & .122 & & $1.036(.991-1.082)$ \\
\hline \multirow[t]{7}{*}{ Kujala } & Sex & .085 & .022 & $6.624(1.307-33.573)$ & .102 & & $3.615(.775-16.866)$ \\
\hline & Age & .141 & & $.964(.919-1.012)$ & .249 & & $.966(.911-1.024)$ \\
\hline & BMI & .018 & .330 & $.955(.871-1.047)$ & .049 & .209 & $.946(.867-1.032)$ \\
\hline & WC & .461 & & $.528(.096-2.889)$ & .706 & & $.655(.073-5.875)$ \\
\hline & Age at first instability event & .039 & .539 & $.974(.896-1.059)$ & .624 & & $.984(.923-1.049)$ \\
\hline & Number of instability events & .771 & & $.996(.968-1.025)$ & .702 & & $.993(.961-1.027)$ \\
\hline & Preoperative PRO score & .004 & .096 & $1.032(.994-1.070)$ & .017 & .059 & $1.035(.999-1.072)$ \\
\hline
\end{tabular}

( ise corresponds to univariate regression. OR, odds ratio; 95\% CI, 95\% confidence interval; PASS, patient mentation Committee; KOOS, Knee Injury and Osteoarthritis Outcome Score; ADL, activities of daily living; QOL, quality of life; JR, joint replacement; BMI, body mass index; WC, workers' compensation; PRO, patient-reported outcome. 